\title{
P53 Polymorphism at Codon 72 is Associated with Keratocystic Odontogenic Tumors in the Thai Population
}

\author{
Pattamawadee Yanatatsaneejit $^{1}$, Ajaree Boonsrang ${ }^{1}$, Apiwat Mutirangura ${ }^{2}$, \\ Vyomesh Patel ${ }^{3}$, Nakarin Kitkumthorn ${ }^{4 *}$
}

\begin{abstract}
Objective: To clarify the association between the $p 53$ polymorphism at codon 72 and susceptibility to the sporadic keratocystic odontogenic tumor (KCOT). Design: One hundred KCOTs and 160 match-group healthy controls were genotyped to ascertain the frequency of the $p 53$ codon 72 polymorphism using polymerase chain reaction-restriction fragment length polymorphism (PCR-RFLP), confirmed by direct sequencing. frequencies of the Pro/Pro, Arg/Pro, and Arg/Arg genotypes were 23.8\%, 49.4\%, and 26.9\%, respectively, in the controls, while the KCOT cohort demonstrated $43.0 \%, 39.0 \%$, and $18.0 \%$, respectively. Further analysis suggested that $p 53$ Pro could be a KCOT-susceptible allele $(\mathrm{OR}(95 \% \mathrm{CI})=1.77(1.22$ to 2.59$), p=0.0024)$, with a sex-adjusted OR $(95 \% \mathrm{CI})$ of $1.71(\mathbf{1 . 1 7 - 2 . 5 0}), \boldsymbol{p}=\mathbf{0 . 0 0 4 6}$. Moreover, the results indicated that $p 53$ codon 72 Pro homozygous was associated with a two-fold risk of developing KCOT (adjusted OR $(95 \% \mathrm{CI})=\mathbf{2 . 1 7}(\mathbf{1 . 2 3 - 3 . 8 4})$, $\mathbf{p = 0 . 0 0 6 2 )}$. Conclusions: The $\mathrm{C} / \mathrm{C}$ genotype of $P 53$ gene codon 72 increases the risk of developing sporadic KCOT and may be a useful tool for screening and diagnostic purposes.
\end{abstract}

Keywords: Keratocystic odontogenic tumor - P53 - polymorphism - risk

Asian Pac J Cancer Prev, 16 (5), 1997-2001

\section{Introduction}

Keratocystic odontogenic tumor (KCOT) represents a common benign lesion of primarily odontogenic origin that arise from the dental lamina and its remnants. It is a rare and benign but develops into a locally aggressive cystic neoplasia. Histopathologically, KCOT displays typical feature of corrugated and thin parakeratinized stratified squamous epithelium lining the cystic wall (Philipsen and Reichart, 2006; Bhargava et al., 2012). Furthermore, KCOT lesions are usually classified as sporadic or those associated with nevoid basal cell carcinoma syndrome (NBCCS) that mainly comprises multiple basal cell carcinomas of skin, calcification of falx cerebri, rib and vertebrate anomalies and KCOT (Philipsen and Reichart, 2006). Nonetheless, what remains crucial is that KCOT can manifest either as asymptomatic or as aggressive infiltrating lesions with a high recurrent rate. A challenge though, is that underlying molecular basis of this variable behavior remains unclear and an area of interest for further investigation. Research activities from several groups have now reported the involvement of key important molecules that may be playing a role in the pathogenesis of KCOT. For example, a study by Barreto, et al found a 5 bp deletion in exon 3 in 1 of 3 sporadic cyst and nonsense (C2760A) and missense (G3499A) mutation in 2 of 3 cyst associated with NBCCS (Barreto et al., 2000). A study by Guo, et al showed 11 germline and 4 somatic cell mutation of $P T C H$ in 11 of 14 patients with NBCCS associated with KCOT (Guo et al., 2013). In addition, Moreira, et al, detected $p 21$ promoter methylation in 3 of $10 \mathrm{KCOT}$ samples whereas no methylation could be detected in 10 samples of dental follicle and 6 samples of normal mucosa (Moreira et al., 2009). Moreover, work from our group has demonstrated that levels of methylation are essentially different in KCOT and ameloblastoma (Kitkumthorn and Mutirangura, 2010). Notably, these observed differences between the two sample groups were irrespective of clinical symptom and radiographic features which alone are largely inseparable. In this regard, a definitive diagnosis is essentially determined by a pathologist, after reviewing histopathological $\mathrm{H} \& \mathrm{E}$ stained tissue sections, however subtle differences between KCOT samples may be missed.

The p53 tumor suppressor gene plays a crucial role in modulating key cellular processes such as cell cycle checkpoint, cell cycle arrest and apoptosis (Hanahan and Weinberg, 2011). Invariably, the $p 53$ gene is a frequent

${ }^{1}$ Human Genetics Research Group, Department of Botany, Faculty of Science, ${ }^{2}$ Center of Excellence in Molecular Genetics of Cancer and Human Diseases, Department of Anatomy, Faculty of Medicine, Chulalongkorn University, ${ }^{4}$ Department of Oral and Maxillofacial Pathology, Faculty of Dentistry, Mahidol University, Bangkok, Thailand, ${ }^{3}$ Cancer Research Initiatives Foundation (CARIF), 2nd Floor Outpatient Centre, Sime Darby Medical Centre, 47500 Subang Jaya, Malaysia*For correspondence: Nakarinkit@gmail.com 
target for alterations, for example, deletions, frame shift, missense, nonsense and promoter methylation resulting in loss of function and tumorigenesis (Chen et al., 1994; Rivlin et al., 2011; Chmelarova et al., 2013). Moreover, p53 single nucleotide polymorphism (SNP) at codon 72 , has also been associated as a risk factor in many precancers and cancer including those of oral lichen planus (Yanatatsaneeji et al., 2010), cancer of cervical (Zhou et al., 2012), colorectal (Aizat et al., 2011), lung (Ren et al., 2013), breast (Vijayaraman et al., 2012) and bladder (Xu et al., 2012). In this regard, two forms of this $p 53$ SNP, Arg and Pro are now recognized as playing a role in tumorigenesis and notably in patients with differing demographics. For example, recent studies have found that Arg is likely to be associated with colorectal and breast cancers in Greek patient population (Kalemi et al., 2005; Dakouras et al., 2008), while largely prevalent in cervical cancer patients of Sudanese origin (Eltahir et al., 2012). The Pro SNP on the other hand, was noted to be more common in colorectal cancer patients of Malaysian (Aizat et al., 2011) and Chinese origin (Zhu et al., 2007), and lung cancer patients of Korean origin (Piao et al., 2011). In this light, follow up studies from our group observed that $p 53$ codon 72 polymorphism was closely aligned with ameloblastoma and suggested that this alteration could be the risk factor of this disease (Kitkumthorn et al., 2010).

Thus the focus of this study was aimed to evaluate if there was any indication of an association between p53 codon 72 polymorphism and the risk of developing sporadic KCOT in a Thai patient population. Consequently, any new emerging knowledge can likely help in our understanding of the molecular basis of KCOT as well as identifying new strategies to help different forms of KCOT and help to distinguish between KCOT and ameloblastoma lesions, with the overall view of facilitating the overall diagnosis to aide in the choice of correct treatment options.

\section{Materials and Methods}

\section{Sample collection and DNA extraction}

One hundred (100) formalin fixed and paraffinembedded (FFPE) and histopathologically confirmed KCOT biopsies collected and archived between January 2006 and December 2010 at the Department of Oral and Maxillofacial Pathology, Faculty of Dentistry, Mahidol University, were used in this study.

All specimens further underwent histopathological assessment by an oral pathologist, for reconfirmation of the presence of KCOT. In parallel, clinical data for each case was collected from archived patients' charts. Healthy controls undergoing routine health examination and found to have no past history of malignancies were enrolled into this study. Six milliliter of peripheral blood was collected from each of the 160 healthy controls after obtaining written informed consent. Both the KCOT and healthy control patient cohorts were of Thai demographics. This arm of the experiment design was approved by the Human Research Ethics Committee of Chulalongkorn University (IRB no 94/54). This study was done using a double-blind technique. In addition to obtaining sections for H\&E staining, FFPE KCOT tissues were used to obtain $\sim 5 \mu \mathrm{M}$ sections in eppendorf tubes (3-5 sections) which subsequently underwent lysis followed by DNA isolation and purification using the MagneSil ${ }^{\circledR}$ Genomic fixed tissue system kit (Promega, Madison WI, USA). DNA from peripheral blood of each of the healthy subject was extracted by first digesting with proteinase $\mathrm{K}$ and an overnight incubation at $50^{\circ} \mathrm{C}$ followed by phenol/ chloroform extraction and ethanol precipitation. After washing in $70 \%$ ethanol and suspending in $\mathrm{ddH} 2 \mathrm{O}$ the genomic DNA was quantified and 100 nanogram used as a template in genotyping analysis.

\section{P53 genotyping analysis}

Polymorphisms p53 at codon 72, were determined using a polymerase chain reaction-restriction fragment length polymorphism (PCR-RFLP) method using primers (5'CCCGGACGATATTGAACA3' and 5' AGAAGCCCAGACGGAAAC 3'). PCR conditions include denaturation for $15 \mathrm{~min}$ at $95^{\circ} \mathrm{C}$, followed by 35 cycles of $1 \mathrm{~min}$ at $95^{\circ} \mathrm{C}, 1 \mathrm{~min}$ at $57^{\circ} \mathrm{C}$, and $1 \mathrm{~min}$ at $72^{\circ} \mathrm{C}$, and with a final extension for $7 \mathrm{~min}$ at $72^{\circ} \mathrm{C}$. A 10 $\mu \mathrm{L}$ aliquot of the PCR product was digested overnight at $37^{\circ} \mathrm{C}$ in a $15 \mu \mathrm{L}$ reaction volume containing 10 units of $B s t U I$ (New England BioLabs, Ipswich MA). After, DNA fragments were separated by electrophoresis using a $2.5 \%$ agarose gel and after staining with ethidium bromide the bands are visualized. The premise of the band size is based on the fact that Arg/Arg homozygote cleaved by BstUI and essentially yielding two size bands of 125 and $79 \mathrm{bp}$. On the other hand, the Pro/Pro homozygote is resistant to cleavage by $B s t U I$ and represented by a single band of 204 bp, whereas the Arg/Pro heterozygote contained all three bands $(204,125$, and $79 \mathrm{bp})$ following digestion. Twenty percent of samples were confirmed by direct sequencing of PCR products to verify the accuracy of the genotyping.

\section{Statistical analysis}

Genotype frequencies were checked for consistency among normal controls with those expected from the Hardy-Weinberg equilibrium (HWE). All statistical analysis was calculated by using Statcalc Program (AcaStat Software, Leesburg, VA, USA). The correlation between genotype and KCOT was analyzed by chi-square test. A value of $<0.05$ was considered significant. ODDS ratio $(\mathrm{OR})$ and $95 \%$ confidence interval $(\mathrm{CI})$ were used as parameters to compare the frequency of SNP to be a risk factor in KCOT. If OR is $>1$, this indicates a positive association or increase risk between the SNP allele and KCOT and by contrast, $<1$ is referred to a decrease susceptibility to disease outcome at the $95 \%$ confidence interval.

\section{Results}

\section{Demographics}

All 160 healthy controls and $100 \mathrm{KCOT}$ patients were Thai population. Average age of healthy control was 32.51 years (male 31.92 years and female 33.05 years). Average age of the KCOT cohort was 32.23 years (male 35.46 and female 29 years) as shown in Table 1. Patients with KCOT in this study were all sporadic cases. 
Genotyping of SNP: P53 codon 72

Paraffin-embedded KCOT samples and healthy control blood samples were used as specimen for genotyping. The genotype of $p 53$ codon 72 in KCOT and control samples was analyzed after performing PCR-RFLP. From the analysis, three genotype groups of $p 53$ codon 72 in patients with KCOT and control blood samples were detected. As shown in Table 1, genotypes Pro/Pro, Arg/Pro and Arg/ Arg were $23.75 \%, 49.37 \%$ and $26.88 \%$, respectively in controls. While in KCOT samples, this genotype was $43 \%, 39 \%$ and $18 \%$, respectively. The distribution of the genotype of this SNP among the control was in Hardy-Weinberg equilibrium ( $>0.05$ ). An additional 39 heterozygous samples KCOT samples were tested for \% density of 3 PCR to ensure that there was no mosaicism from epithelium cells and the $\% \mathrm{CV}$ of all bands were less than $10 \%$.

\section{SNP analysis of the P53 gene at codon 72 in KCOT}

The emerging data and allele frequency between KCOT and healthy controls is shown in Table 1. These observations were used to perform detailed statistical analysis. From the analysis, we observed that there was a significant risk associated with the $p 53$ codon 72 SNP. The OR (95\% CI) was 1.77 (1.22-2.59) among KCOT cases with $\mathrm{C}$ as the susceptibility alleles. The OR $(95 \%$ $\mathrm{CI})$ in male was $1.39(0.82-1.38)$ and in female this was $2.13(1.22-3.71)$ and sex adjusted, this value came out as $1.71(1.17-2.5)$.

\section{Model of inheritance}

Next, we sought to determine the model of inheritance. From this analysis, we noted that when the model of inheritance of this SNP was recessive, the OR $(95 \% \mathrm{CI})$
P53 Polymorphism and Risk of Keratocystic Odontogenic Tumor of CC was 2.42 (1.37-4.30), sex adjusted was 2.17 (1.233.84). When the mode of inheritance was dominant, the OR (95\% CI) of CC or CG was 1.67 (0.87-3.26), sex adjusted was 1.68 (0.87-3.28). The data is shown in Table 2. Collectively, the data from this analysis suggest that the model of inheritance of p53 codon 72 in KCOT, is recessive.

\section{Discussion}

Single nucleotide polymorphism identified at codon 72 of the $p 53$ gene results in an aberrant amino acid sequence and the varients, Arg and Pro translate into a protein that exhibit very different biochemical properties. For example, Arg is an amino acid with a positive charge and basic- $\mathrm{R}$ group whereas Pro is non-polar and cyclic amino acid (Thomas et al., 1999). Notably, the protein resulting from the Arg variant is more potent than the Pro form in its tumor suppressive properties (Dumont et al., 2003). In addition, the Arg form has now been reported to be more efficient in inducing apoptosis than Pro counterpart. Notwithstanding, cancer is a multistep process with an aberrant $p 53$ playing a pivotal role but whether the SNP at codon 72 of this gene, is involved in carcinogenesis still remains to be determined. In some studies, Pro/Pro and Arg/Arg variants have been found be risk factors for same type of cancers such as lung and breast cancer (Papadakis et al., 2000; Buyru et al., 2008; Piao et al., 2011; Proestling et al., 2012). Perhaps pertinent, frequencies of $p 53$ codon 72 genotypes have been postulated to vary according to ethnicity (Xu et al., 2012). Thus, this emerging data suggests that the codon 72 alleles of p53 might not to be functionally involved in cancer but, rather, may be a marker in linkage disequilibrium. However, more studies

Table 1. P53 Codon 72 Polymorphism in KCOTs and Controls

\begin{tabular}{|c|c|c|c|c|c|c|c|c|c|}
\hline \multirow[t]{2}{*}{ Group } & \multirow{2}{*}{$\begin{array}{c}\text { No. of } \\
\text { samples }\end{array}$} & \multirow{2}{*}{$\begin{array}{c}\text { Ave. } \\
\text { age (y.) }\end{array}$} & \multicolumn{3}{|c|}{ P53 genotype $[\mathrm{n}(\%)]$} & \multicolumn{2}{|c|}{ Allele frequency $[\mathrm{n}(\%)]$} & \multirow{2}{*}{$\begin{array}{c}\text { Allelic test } \\
\text { OR }(95 \% \text { CI })\end{array}$} & \multirow[t]{2}{*}{$P$-value } \\
\hline & & & $\begin{array}{l}\text { Pro } \\
\mathrm{C} / \mathrm{C}\end{array}$ & $\begin{array}{l}\text { Hetero } \\
\mathrm{C} / \mathrm{G}\end{array}$ & $\begin{array}{c}\text { Arg } \\
\text { G/G }\end{array}$ & $\mathrm{C}$ & G & & \\
\hline Controls & 160 & 32.51 & $38(23.75 \%)$ & $79(49.37 \%)$ & $43(26.88 \%)$ & $155(48.44 \%)$ & $165(51.56 \%)$ & Ref. & \\
\hline \multicolumn{10}{|c|}{ 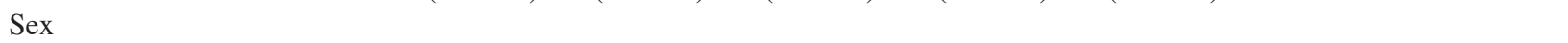 } \\
\hline Male & $80(50.00 \%)$ & 31.92 & $20(25.00 \%)$ & $38(47.50 \%)$ & $22(27.50 \%)$ & $78(48.75 \%)$ & $82(51.25 \%)$ & Ref. & \\
\hline Female & $80(50.00 \%)$ & 33.05 & $21(26.25 \%)$ & $38(47.50 \%)$ & $21(26.25 \%)$ & $80(50.00 \%)$ & $80(50.00 \%)$ & Ref. & \\
\hline KCOT & 100 & 32.23 & $43(43.00 \%)$ & $39(39.00 \%)$ & $18(18.00 \%)$ & $125(62.50 \%)$ & $75(37.50 \%)$ & $1.77(1.22-2.59)$ & 0.0024 \\
\hline \multicolumn{10}{|l|}{ Sex } \\
\hline Male & $50(50.00 \%)$ & 35.46 & $20(40.00 \%)$ & $17(34.00 \%)$ & $13(26.00 \%)$ & $57(57.00$ & $43(43.00 \%)$ & $.82-2.38)$ & 0.2429 \\
\hline Female & $50(50.00 \%)$ & 29 & $23(46.00 \%)$ & $22(44.00 \%)$ & $5(10.00 \%)$ & $68(68.00 \%)$ & $32(32.00 \%)$ & $2.13(1.22-3.71)$ & 0.0065 \\
\hline \multicolumn{3}{|c|}{ Adjust OR } & & & & & & $1.71(1.17-2.50)$ & 0.0046 \\
\hline
\end{tabular}

OR; odd ratio, $\mathrm{CI}$; confidence interval

Table 2. Risk of KCOT Associated with P53 Codon 72 Genotype according to Different Models of Inheritance

\begin{tabular}{|c|c|c|c|c|c|c|c|c|}
\hline & $\begin{array}{c}\text { KCOT } \\
\text { OR }(95 \% \mathrm{CI})\end{array}$ & $P$-value & $\begin{array}{c}\text { Male } \\
\text { OR }(95 \% \mathrm{CI})\end{array}$ & $P$-value & $\begin{array}{c}\text { Female } \\
\text { OR }(95 \% \mathrm{CI})\end{array}$ & $P$-value & $\begin{array}{c}\text { Adjusted OR } \\
(95 \% \mathrm{CI})\end{array}$ & $P$-value \\
\hline \multicolumn{9}{|c|}{$\mathrm{C}$ dominance, $\mathrm{G}$ wild type } \\
\hline GG & 1.00 & & 1.00 & & 1.00 & & 1.00 & \\
\hline $\mathrm{CC}$ or $\mathrm{CG}$ & $1.67(0.87-3.26)$ & 0.1356 & $1.08(0.45-2.60)$ & 0.9875 & $1.97(0.86-4.51)$ & 0.1184 & $1.68(0.87-3.28)$ & 0.1358 \\
\hline \multicolumn{9}{|c|}{$\mathrm{C}$ recessive, $\mathrm{G}$ wild type } \\
\hline $\mathrm{CG}$ or $\mathrm{GG}$ & 1.00 & & 1.00 & & 1.00 & & 1.00 & \\
\hline $\mathrm{CC}$ & $2.42(1.37-4.30)$ & 0.0018 & $3.20(1.03-10.57)$ & 0.0425 & $2.39(1.06-5.41)$ & 0.0336 & $2.17(1.23-3.84)$ & 0.0062 \\
\hline
\end{tabular}

*CC and GG are the homozygous $\mathrm{C}$ and $\mathrm{G}$, respectively. $\mathrm{CG}$ is the heterozygous. OR(95\%) is odd ratios and $95 \%$ interval between allele and compared allele; *Fisher exact test: 1 tailed test 
are needed to definitively define a role of codon 72 alleles of p53 with the multistep process of cancer including KCOT.

The molecular approach for investigating KCOT and specifically to distinguish the different forms that could be missed by histopathological evaluation is in need. In this regard, molecular evaluation could be a useful addition for clinical diagnosis, prevention and treatment. A number of genetic based studies have shown a role for PTCH and p21WAF1 in the etiology of KCOT (Barreto et al., 2000; Moreira et al., 2009; Guo et al., 2013). A study by Barreto, et al suggested that mutations in PTCH could be an important first step in KCOT (Barreto et al., 2000). A study by Guo YY et al, 2013, reported that PTCH was mutated in KCOT in Chinese patients (Guo et al., 2013). While the study by Moreira, et al. reported that the $p 21 W A F 1$ promoter was methylated in 3 of $10 \mathrm{KCOT}$ samples whereas no methylation could be detected in 10 dental follicles and 6 normal mucosa samples (Moreira et al., 2009).

In this study, we addressed whether an association of p53 codon 72 alleles with KCOT was present in a Thai patient population. From the emerging data, we observed that the genotype Pro/Pro (proline) was estimated to have a 2.17 fold increased risk to developing KCOT compared to normal controls. In addition, we also noted that the mode of inheritance of this SNP was recessive. From these observations, we report that the Pro/Pro genotype is a risk factor for KCOT in Thai patient population. Notably, our previous study also found that there was a significant association between the Arg/Arg (arginine) genotype of p53 and a prominent risk for ameloblastoma (Kitkumthorn et al., 2010). This difference, where the arginine and proline isoforms increases risk for ameloblastoma and $\mathrm{KCOT}$, respectively, is likely due to different biological function of these variants.

In conclusion, we present emerging data from our current study that furthers our understanding of molecular basis of KCOT and when combined with observation from our previous study, genotyping $p 53$ codon 72 in both KCOT and ameloblastoma may discriminate these two lesions with have similar clinical symptoms and histopathological and radiographical features making diagnosis very challenging. Our molecular approach offers a simple and a relatively efficient approach to investigate KCOT and ameloblastoma for accurate diagnosis in the present.

\section{Acknowledgements}

This research has been supported by the Ratchadapiseksomphot Endowment Fund of Chulalongkorn University (CU-57-001-HR).

\section{References}

Aizat AA, Shahpudin SN, Mustapha MA, et al (2011). Association of Arg72Pro of P53 polymorphism with colorectal cancer susceptibility risk in Malaysian population. Asian Pac J Cancer Prev, 12, 2909-13.

Barreto DC, Gomez RS, Bale AE, et al (2000). PTCH gene mutations in odontogenic keratocysts. J Dent Res, 79, 1418-22.

Bhargava D, Deshpande A and Pogrel MA, (2012). Keratocystic odontogenic tumour (KCOT)--a cyst to a tumour. Oral Maxillofac Surg, 16, 163-70.

Buyru N, Altinisik J, Isin M, et al (2008). p53 codon 72 polymorphism and HPV status in lung cancer. Med Sci Monit, 14, 493-7.

Chen Y, Xu L, Massey L, et al (1994). Frameshift and nonsense p53 mutations in squamous-cell carcinoma of head and neck - non-reactivity with 3 anti-p53 monoclonal-antibodies. Int J Oncol, 4, 609-14.

Chmelarova M, Krepinska E, Spacek J, et al (2013). Methylation in the $p 53$ promoter in epithelial ovarian cancer. Clin Transl Oncol, 15, 160-3.

Dakouras A, Nikiteas N, Papadakis E, et al (2008). P53Arg72 homozygosity and its increased incidence in left-sided sporadic colorectal adenocarcinomas, in a Greek-Caucasian population. Anticancer Res, 28, 1039-43.

Dumont P, Leu JI, Della Pietra AC, 3rd, et al (2003). The codon 72 polymorphic variants of $p 53$ have markedly different apoptotic potential. Nat Genet, 33, 357-65.

Eltahir HA, Elhassan AM and Ibrahim ME, (2012). Contribution of retinoblastoma $\mathrm{LOH}$ and the $p 53 \mathrm{Arg} /$ Pro polymorphism to cervical cancer. Mol Med Rep, 6, 473-6.

Guo YY, Zhang JY, Li XF, et al (2013). PTCH1 gene mutations in Keratocystic odontogenic tumors: a study of 43 Chinese patients and a systematic review. PLoS One, $8,77305$.

Hanahan D and Weinberg RA, (2011). Hallmarks of cancer: the next generation. Cell, 144, 646-74.

Kalemi TG, Lambropoulos AF, Gueorguiev M, et al (2005). The association of $p 53$ mutations and $p 53$ codon 72 , Her 2 codon 655 and MTHFR C677T polymorphisms with breast cancer in Northern Greece. Cancer Lett, 222, 57-65.

Kitkumthorn N and Mutirangura A, (2010). LINE-1 methylation difference between ameloblastoma and keratocystic odontogenic tumor. Oral Dis, 16, 286-91.

Kitkumthorn N, Yanatatsaneejit P, Rabalert J, et al (2010). Association of P53 codon 72 polymorphism and ameloblastoma. Oral Dis, 16, 631-5.

Moreira PR, Guimaraes MM, Guimaraes AL, et al (2009). Methylation of P16, P21, P27, RB1 and P53 genes in odontogenic keratocysts. J Oral Pathol Med, 38, 99-103.

Papadakis EN, Dokianakis DN and Spandidos DA, (2000). p53 codon 72 polymorphism as a risk factor in the development of breast cancer. Mol Cell Biol Res Commun, 3, 389-92.

Philipsen HP and Reichart PA, (2006). Classification of odontogenic tumours. A historical review. J Oral Pathol Med, 35, 525-9.

Piao JM, Kim HN, Song HR, et al (2011). p53 codon 72 polymorphism and the risk of lung cancer in a Korean population. Lung Cancer, 73, 264-7.

Proestling K, Hebar A, Pruckner N, et al (2012). The Pro allele of the $p 53$ codon 72 polymorphism is associated with decreased intratumoral expression of BAX and $\mathrm{p} 21$, and increased breast cancer risk. PLoS One, 7, 47325 .

Ren YW, Yin ZH, Wan Y, et al (2013). P53 Arg72Pro and MDM2 SNP309 polymorphisms cooperate to increase lung adenocarcinoma risk in Chinese female non-smokers: a case control study. Asian Pac J Cancer Prev, 14, 5415-20.

Rivlin N, Brosh R, Oren M, et al (2011). Mutations in the $p 53$ tumor suppressor gene: important milestones at the various steps of tumorigenesis. Genes Cancer, 2, 466-74.

Thomas M, Kalita A, Labrecque S, et al (1999). Two polymorphic variants of wild-type $p 53$ differ biochemically and biologically. Mol Cell Biol, 19, 1092-100.

Vijayaraman KP, Veluchamy M, Murugesan P, et al (2012). 
p53 exon 4 (codon 72) polymorphism and exon 7 (codon 249) mutation in breast cancer patients in southern region (Madurai) of Tamil Nadu. Asian Pac J Cancer Prev, 13, 511-6.

Xu T, Xu ZC, Zou Q, et al (2012). P53 Arg72Pro polymorphism and bladder cancer risk--meta-analysis evidence for a link in Asians but not Caucasians. Asian Pac J Cancer Prev 13, 2349-54.

Yanatatsaneeji P, Kitkumthorn N, Dhammawipark C, et al (2010). Codon 72 polymorphism in the $p 53$ tumor suppressor gene in oral lichen planus lesions in a Thai population. Asian Pac J Cancer Prev, 11, 1137-41.

Zhou X, Gu Y and Zhang SL (2012). Association between $p 53$ codon 72 polymorphism and cervical cancer risk among Asians: a HuGE review and meta-analysis. Asian Pac J Cancer Prev, 13, 4909-14.

Zhu ZZ, Wang AZ, Jia HR, et al (2007). Association of the TP53 codon 72 polymorphism with colorectal cancer in a Chinese population. Jpn J Clin Oncol, 37, 385-90. 